\title{
Expression Systems for Soluble Metal-Dependent Formate Dehydrogenase
}

Masaki Ihara ${ }^{1,2 *}$, Yusuke Kawano ${ }^{3}$, Yusuke Fujiwara ${ }^{1}$, Tetsuya Kodo $^{1}$, Manami Mizuguchi ${ }^{1}$, Keisuke Mochiduki $^{1}$, Kai Kodoh ${ }^{1}$, Ayako Okabe ${ }^{1}$, Izumi Matsuno ${ }^{1}$

Faculty of Agriculture, Shinshu University, 8304 Minamiminowa, Nagano 399-4511, Japan, ${ }^{1}$ JST, PRESTO, 4-1-8 Honcho, Kawaguchi, Saitama 332-0012, Japan, ${ }^{2}$ and Graduate School of Biological Sciences, Nara Institute of Science and Technology, 8916-5 Takayama, Ikoma, Nara 630-0192, Japan ${ }^{3}$

*Corresponding author.

Telephone/Fax: +81-265-77-0508, email address: m_ihara@shinshu-u.ac.jp

\begin{abstract}
Molybdenum or tungsten-dependent formate dehydrogenases $(\mathrm{FDH})$ can reduce carbon dioxide $\left(\mathrm{CO}_{2}\right)$ to formate under ordinary conditions, and therefore, are considered promising catalysts for $\mathrm{CO}_{2}$ fixation. However, to our knowledge, no study on the modification of metal-dependent FDHs has been published, likely because of a lack of convenient expression systems for the recombinant enzymes. We attempted to establish an methodology for the preparation and modification of soluble oxygen-tolerant metal-dependent FDHs, for the following three strategies: (1) Escherichia coli FDH is converted from a membrane bound protein into a soluble protein by deleting the C-terminal membrane-anchor of small subunit $(\mathrm{FdoH})$ and the whole membrane subunit (FdoI) and expressed homologously in E. coli; (2) originally soluble FDHs from Desulfovibrio are expressed heterologously in E. coli; and (3) Desulfovibrio FDHs are genetically engineered by the homologous gene recombination method and expressed homologously in Desulfovibrio. We successfully established the expression systems for (1) and (3), and succeeded in purification of the soluble FDHs with a tag by using affinity columns.
\end{abstract}

\section{Introduction}

1 Formate dehydrogenase (FDH) is an enzyme that can reduce carbon dioxide $\left(\mathrm{CO}_{2}\right)$ to formate. Two classes 2 of FDHs have been discovered thus far: metal-independent nicotinamide adenine dinucleotide (NADH)3 based FDHs [1, 2] and metal-dependent molybdenum (Mo)-based or tungsten (W)-based FDHs [3-5]. We 
had earlier created several NADPH-dependent type FDHs and combined them with photosystem I (PSI), ferredoxin, and ferredoxin-NADP ${ }^{+}$-reductase to establish an artificial light-driven carbon dioxide reduction system (Figure 1A). The system successfully produced formate in vitro and in vivo [6]. However, the reduction of carbon dioxide by NADPH $\left(\mathrm{CO}_{2}+\mathrm{NADPH} \rightarrow \mathrm{HCOO}^{-}+\mathrm{NADP}^{+}\right)$is thermodynamically unfavorable. To create a more efficient system, it is necessary to use a metal-dependent FDH that can directly accept electrons from a low potential electron donor, such as PSI (Figure 1B). Metal-dependent FDHs consist of a catalytic subunit and electron transfer subunits. The catalytic subunit possesses a Mo or $\mathrm{W}$ coordinated by a selenocysteine (SeCys) residue, two molybdopterin guanine dinucleotides (MGDs), and [4Fe-4S] clusters [7-10]. Escherichia coli has three metal-dependent FDHs; formate dehydrogenase $\mathrm{H}(\mathrm{EcFDH}-\mathrm{H})$, formate dehydrogenase $\mathrm{N}(\mathrm{EcFDH}-\mathrm{N})$ and formate dehydrogenase $\mathrm{O}$ (EcFDH-O). EcFDH-H is a part of the multifunctional membrane-bound protein, the formate hydrogenlyase complex, responsible for the anaerobic oxidation of formate to $\mathrm{CO}_{2}$ and molecular hydrogen. It can be isolated as a soluble monomeric enzyme [7, 11-13]. EcFDH-N is a component of an anaerobic respiratory chain, formate-nitrate oxidoreductase, which is also a membrane-bound protein complex and catalyzes oxidation of formate coupled to nitrate respiration [14]. It was purified and crystallized as a trimeric enzyme containing a catalytic subunit $(\mathrm{FdnG})$, an electron transfer subunit $(\mathrm{FdnH})$, and a transmembrane subunit with type-b heme (FdnI) (Figure 2A). EcFDH-O, an isoenzyme of EcFDH-N, is a membrane-bound enzyme composed of three subunits, FdoG, FdoH, and FdoI, which exhibit high sequence similarity to the corresponding subunits of EcFDH-N. The expression of EcFDH-O is induced under micro-aerobic and aerobic conditions. It is proposed that a physiological role of EcFDH-O is to ensure rapid adaptation during a 24 sudden shift from aerobiosis to anaerobiosis [15]. The dimer of FdoG and FdoH is directed toward the cytoplasmic side, and with FdoH anchored to the periplasmic membrane and the FdoI subunit by a Cterminal hydrophobic domain [16]. Among these three metal-dependent FDHs from E. coli, EcFDH-H has been well-studied due to its structural simplicity and solubility [3], but it is intractable for practical applications due to its extremely high oxygen-sensitivity.

In contrast to E. coli FDHs, the metal-dependent FDHs from some anaerobic bacteria, such as sulfate reducing bacteria and Syntrophobacter fumaroxidans, are expressed in a soluble form and are thought to transfer electrons via several types of periplasmic electron carrier proteins by forming a complex in the 
periplasmic space $[4,17-19]$. Their subunit structures are relatively simple, containing a catalytic subunit and one or two electron transfer subunit(s) (Figure 2B). Therefore, they are considered suitable for

34 bioengineering applications and have been used as catalysts for $\mathrm{CO}_{2} /$ formate interconversion on electrodes $[4,20]$. However, the electron transfer between the FDHs and electrodes remains to be improved. In addition, the FDHs are easily inactivated, which is a critical obstacle for practical applications. The manipulation of various properties and stability of enzymes is usually accomplished by alteration of the amino acid sequence. However, such protein engineering attempts have not been carried out because of the difficulty in gene-manipulation of anaerobic bacteria and lack of heterologous expression systems.

To overcome these setbacks with regard to metal-dependent FDHs, we tried to establish expression systems for the recombinant soluble enzymes. For our expression systems, we chose to produce EcFDH-O and metaldependent FDHs from Desulfovibrio vulgaris Miyazaki F and Desulfovibrio gigas, since they are oxygentolerant and can be purified under aerobic conditions. We adopted three strategies: (1) Conversion of

44 EcFDH-O into a soluble protein by deleting the C-terminal membrane-anchor of FdoH and the FdoI subunit and homologous expression in E. coli; (2) Heterologous expression of the soluble FDHs from Desulfovibrio in E. coli; and (3) Homologous expression of the modified Desulfovibrio FDHs in genetically engineered (by

47 homologous gene recombination) D. vulgaris.

Materials and methods

\section{Strain, Culture medium and growth conditions}

51 All bacterial strains used in this study are listed in Table 1. D. vulgaris (DSM19637) and D. gigas (DSM1382) were purchased from Leibniz-Institut DSMZ-German Collection of Microorganisms and Cell Cultures. E. coli strains, BW25113 and the derivatives, JW3865 $\left(\Delta f d o G:: K_{a n}^{R}\right)$ and JW3863 $\left(\Delta f d o I:: \operatorname{Kan}^{R}\right)$, were obtained from E. coli Keio Knockout Collection. The cultivation of D. vulgaris in liquid medium was performed under anaerobic conditions as described previously [21]. The liquid medium contained $0.5 \mathrm{~g} \mathrm{~K}_{2} \mathrm{HPO}_{4}, 2.0 \mathrm{~g} \mathrm{MgSO}_{4} 7 \mathrm{H}_{2} \mathrm{O}, 1.0 \mathrm{~g} \mathrm{Na}_{2} \mathrm{SO}_{4}, 1.0 \mathrm{~g} \mathrm{NH} 4 \mathrm{Cl}$,

$570.1 \mathrm{~g} \mathrm{CaCl}_{2} 2 \mathrm{H}_{2} \mathrm{O}, 1.0 \mathrm{~g}$ yeast extract, $1.0 \mathrm{mg}$ sodium resazurin, $3.3 \mathrm{~g} 60 \%$ sodium lactate and $0.1 \mathrm{~g} \mathrm{~L}-$ ascorbic acid in $1 \mathrm{~L}$ water, with $\mathrm{pH}$ adjusted to 7.8 using $\mathrm{NaOH}$. For the solid plate culture, the cell suspension and melted top agar were mixed under aerobic conditions and then quickly poured on the bottom 
agar medium. The top agar contained $3.0 \mathrm{~g}$ tryptic soy broth, $7.5 \mathrm{~g}$ agar powder, $2.0 \mathrm{~g} \mathrm{MgSO}_{4} 7 \mathrm{H}_{2} \mathrm{O}, 4.2 \mathrm{~g}$ $60 \%$ sodium lactate, $0.9 \mathrm{~g}\left(\mathrm{NH}_{4}\right)_{2} \mathrm{Fe}\left(\mathrm{SO}_{4}\right)_{2} 12 \mathrm{H}_{2} \mathrm{O}, 0.5 \mathrm{~g}$ sodium resazurin, $0.2 \mathrm{~g}$ sodium thioglycolate and 0.2 $\mathrm{g}$, $\mathrm{L}$-ascorbic acid in $1 \mathrm{~L}$ water, $\mathrm{pH}$ adjusted to 7.8 with $\mathrm{NaOH}$. The composition of bottom agar medium was the same as that of the top agar, except that tryptic soy broth was excluded and $20 \mathrm{~g} / \mathrm{L}$ agar power was added to the medium. The plate cultures inoculated with D. vulgaris were incubated under strict anaerobic conditions at $30^{\circ} \mathrm{C}$.

E. coli was cultivated using LB broth, Lennox. In the microaerobic and aerobic cultures, cells were added to Erlenmeyer flasks filled with LB medium up to $50 \%$ and $10 \%$ of the height, respectively, flasks were loosely capped with aluminum foil and incubated at $30^{\circ} \mathrm{C}$ with shake speeds of 100 and $130 \mathrm{rpm}$, respectively. Deletions in the $f d n G H I$ and $f d o G H I$ genes were introduced into $E$. coli BW25113 strain using temperaturedependent suicide vectors, $\mathrm{pKO} 3-\Delta \mathrm{fdo}$ and $\mathrm{pKO}-\Delta \mathrm{fdn}$, producing the $\mathrm{BW} \Delta \Delta$ strain that harbors deletions in EcFDH-O and EcFDH-N.

When required, ampicillin was added to a final concentration of $100 \mu \mathrm{g} / \mathrm{ml}$, and chloramphenicol to a final concentration of 10 or $30 \mu \mathrm{g} / \mathrm{ml}$ to the cultures of $D$. vulgaris or E. coli, respectively.

\section{Genetic engineering}

The cloning vectors, pUC19, pBR322 and pACYC184, were obtained from TaKaRa Bio (Otsu, Japan). For genetic manipulations, FastDigest restriction enzymes (Thermo Fisher Scientific, Massachusetts, USA), PrimeStar GXL DNA polymerase (TaKaRa Bio) or KOD FX DNA polymerase (TOYOBO, Osaka, Japan) and In-Fusion HD Cloning kit (TaKaRa Bio) were used for the digestion, amplification and ligation of DNA fragments, respectively. The extraction of genomic DNA, plasmid DNA and total RNA were carried out using Genomic DNA Extraction Kit (RBC Bioscience, New Taipei City, Taiwan), Hi Yield Plasmid Mini Kit (RBC Bioscience) and High Pure RNA Isolation Kit ver.12 (Roche Applied Science, Penzberg, Upper Bavaria, Germany). The total RNA samples were reverse transcribed to cDNA using Rever Tra Ace ${ }^{\circledR}$ qPCR RT Master Mix with gDNA remover (TOYOBO) and random hexamer primer. Quantitative PCR was conducted using an ABI Step One (Applied. Biosystems; Carlsbad, CA), with THUNDERBIRD ${ }^{\circledR}$ SYBR $^{\circledR}$ qPCR Mix (TOYOBO). 
89 The primer sequences are listed in Table 2, and the plasmid structures are shown in Figure 3. A temperature dependent suicide plasmid for the fdo gene destruction, pKO3- $\Delta$ fdo, was prepared by combining the NotI digested pKO3 [22] with two DNA fragments containing partial sequences of $f d o G$ and $f d o I$, which were amplified from E. coli genomic DNA using the primer sets 1 and 2, respectively. A plasmid for the $f d n$ gene destruction, $\mathrm{pKO} 3-\Delta \mathrm{fdn}$, was synthesized in the same manner as pKO3- $\Delta \mathrm{fdo}$, using the primer sets 3 and 4 . pBR322 was digested with EcoRI and SalI and inserted with a fragment containing the promoter region of the $f d o$ operon, $f d o G, f d o H$ and $f d o I$, amplified from E. coli genomic DNA with the primer set 5, generating the plasmid pBR-fdoGHI, for the expression of the wild-type membrane bound EcFDH-O. The expression plasmids for the solubilized EcFDH-O-pBR-fdoGH, pBR-fdoGHst and pBR-fdoGHtst, were prepared in the same manner as pBR-fdoGHI, by using the primer sets 6,7 and 8 , respectively, instead of the primer set 5.

pUC-based expression plasmids were prepared as follows. The linearized pUC19 was amplified with the primer set 9 by inverse PCR and ligated to a fragment amplified from pBR-fdoGHst with the primer set 10, resulting in pUC-fdoGHtst. pUC-fdoGHtHis was obtained by inverse PCR using pUC-fdoGHtst as a template, with the primer set 11. pUC-fdoGHtst and pUC-fdoGHtHis contain the gene coding for EcFDH-O, with deletion in the membrane subunit and membrane anchor domain and tagged with Strep-tagII and histidine tag at the C-terminal, respectively.

The plasmids for the expression of D. vulgaris FDHs and D. gigas FDH were prepared using pBR322 or pUC19 and E. coli fdo promoter. pBR322 was digested with EcoRI and SalI and ligated to the E. coli fdo promoter (amplified from E. coli genomic DNA using the primer set 12), and the D. vulgaris FDH genes (DvMF643 and 644) (amplified using the primer set 13), yielding pBR-DvMF643. pBR-DvMF646, pBRDvMF1217, pBR-DvMF2533, pBR-DvMF643st, pBR-DvMF646st, pBR-DvMF1217st, and pBRDvMF2533st were prepared in the same manner as pBR-DvMF643, by using primer sets 14, 15, 16, 17, 18 , 113 digested with EcoRI and SalI, the E. coli fdo promoter, and the D. gigas FDH genes (DgfdhA and DgfdhB) 114 amplified using primer sets 21 and 22. pUC-DvMF1217st and pUC-DgFDHst were obtained using the 
inverse PCR product amplified from pUC19 with the primer set 9, and the fragments amplified from pBR-

DvMF1217st and pBR-DgFDHst, respectively, with the primer set 23.

A plasmid for the chimera FDH, pUC-fdoG/DgigHst, composed of the catalytic subunit of EcFDH-O and the electron transfer subunit of D. gigas FDH, was synthesized by combining the inverse PCR product of pUC19, the fragment amplified from pBR-fdoGH, with the primer set 24 and the fragment amplified from pBR-DgFDHst with the primer set 25 .

121 Plasmids for the co-expression of accessory genes were prepared as follows: pACYC-FdhE645 was prepared 122 by inserting the fragment amplified from E. coli genomic DNA using the primer set 26 into the XbaI and SalI sites of pACYC184. pACYC-FdhE648 and pACYC-FdhDE1233 were prepared in a similar manner as pACYC-FdhE645, by using the primer sets 27 and 28, respectively.

A homologous recombination plasmid pDvMF-cmst was constructed using HindIII-PstI treated pUC19 and four fragments containing a part of the gene for D. vulgaris FDH small subunit, the chloramphenicol cassette, the FDH promoter, and a part of the gene for MobB. The templates and primer sets used to amplify these four fragments were as follows: D. vulgaris genomic DNA with the primer set 29; pACYC184 with the primer set $30 ; D$. vulgaris genomic DNA with the primer set 31 ; and D. vulgaris genomic DNA with the primer set 32. pDvMF-cmPsaE was prepared in the same manner as pDvMF-cmst, using the primer set 33 to amplify the D. vulgaris FDH small subunit, and adding the fragment coding for PsaE gene amplified with the primer set 34 from Synechocystis sp. PCC6803 genome. pDvMF-cmst2 was obtained by inserting a SacB gene amplified with the primer set 35 into pDvMF-cmst digested with $\mathrm{XbaI}$ and $\mathrm{SacI}$.

\section{In-gel visualization of enzyme activity and western blotting}

138 All steps were carried out under aerobic conditions. Cells (50 mg wet weight) were resuspended in $1 \mathrm{ml}$ of $50 \mathrm{mM}$ Tris- $\mathrm{HCl} \mathrm{pH} 7.5$ with $6 \mathrm{U} / \mathrm{ml}$ DNase, $250 \mu \mathrm{g}$ lysozyme, $0.1 \%$ Triton X-100 and $5 \mathrm{mM} \mathrm{MgCl}_{2}$, and incubated for $10 \mathrm{~min}$ at room temperature. Non-denaturing polyacrylamide gel electrophoresis was performed in $7.5 \%$ gel containing $0.1 \%$ Triton $\mathrm{X}-100$ under air atmosphere at $4^{\circ} \mathrm{C}$. After electrophoretic separation of the proteins, the gels were incubated in $50 \mathrm{mM}$ Tris- $\mathrm{HCl} \mathrm{pH} 7.5$ containing $2 \mathrm{mM}$ benzyl 
viologen (BV) and $2 \mathrm{mM}$ nitroblue tetrazolium, $80 \mathrm{mM}$ formate and $0.1 \%$ Triton $\mathrm{X}-100$ under a nitrogen

144 atmosphere at $30^{\circ} \mathrm{C}$. For western blotting, denaturing polyacrylamide gel electrophoresis was performed in $1457.5 \%$ gel containing $0.1 \%$ sodium dodecyl sulfate under air atmosphere at room temperature. The proteins in 146 the gel were then transferred to PVDF membrane and immunostained using anti-NWSHPQFEK (Strep-tagII) 147 antibody (GenScript Inc., Piscataway, NJ) and anti-rabbit IgG antibody (Santa Cruz Biotechnology Inc., 148 Dallas, TX).

\section{Random mutagenesis and screening}

151 Random mutagenesis was performed by amplifying the DNA fragment coding for EcFdoGHt (ca. 4100 bp) using the low fidelity KOD FX DNA polymerase. The DNA fragment was re-inserted into pUC plasmid, and the resulting plasmids were introduced into $\mathrm{BW} \Delta \Delta$, yielding thousands of colonies. Five hundereds randomly selected colonies were cultured in 96-well plates, lysed, and centrifuged. The supernantants were individually added to 8-strip PCR tubes containing StrepTactin-Sepharose beads. After incubation for $10 \mathrm{~min}$ at room temperature, the beads were washed twice with a buffer containing $50 \mathrm{mM}$ Tris- $\mathrm{HCl} \mathrm{pH} 7.5$ and

$0.1 \%$ Triton $\mathrm{X}-100$, and incubated in a buffer containing $50 \mathrm{mM}$ Tris-HCl $\mathrm{pH} 7.5,2 \mathrm{mM}$ benzyl viologen (BV) and $2 \mathrm{mM}$ nitroblue tetrazolium, $80 \mathrm{mM}$ formate, and $0.1 \%$ Triton X-100 under a nitrogen atmosphere at $30^{\circ} \mathrm{C}$.

\section{Protein purification}

162 Cells (10 g wet weight) were resuspended in $50 \mathrm{ml}$ of $50 \mathrm{mM}$ Tris-HCl pH 7.5 with $6 \mathrm{U} / \mathrm{ml}$ DNase, $250 \mu \mathrm{g}$ lysozyme, $0.1 \%$ Triton $\mathrm{X}-100$, and $5 \mathrm{mM} \mathrm{MgCl}$, and incubated for $20 \mathrm{~min}$ at room temperature. After centrifugation, the supernatant was loaded onto a His-accept (Nacalai Tesque, Kyoto, Japan) or a StrepTactin-Sepharose column $(3 \times 2 \mathrm{~cm}$, IBA GmbH, Goettingen, Germany) equilibrated with buffer A (50 $\mathrm{mM}$ Tris- $\mathrm{HCl} \mathrm{pH} 7.5,500 \mathrm{mM} \mathrm{NaCl}$, and $0.1 \%$ Triton X-100). Unbound protein was washed from the column with buffer $\mathrm{A}$, and the protein was then eluted with buffer A containing $500 \mathrm{mM}$ imidazole from the His-accept column or buffer A containing $2.5 \mathrm{mM}$ D-desthiobiotin from the StrepTactin-Sepharose column.

The fractions containing enzyme activity were dialyzed against $50 \mathrm{mM}$ Tris- $\mathrm{HCl} \mathrm{pH} 7.5$ including $0.1 \%$ Triton X-100, and concentrated by centrifugation at 3,500 $\times \mathrm{g}$ in centrifugal filters (Amicon Ultra, $50 \mathrm{~K}$, 
Millipore, Eschborn, Germany). The protein samples were stored at $4{ }^{\circ} \mathrm{C}$ and used within 2 days.

\section{Enzymatic measurement}

FDH activity was routinely determined spectrophotometrically by following the formate-dependent reduction of $\mathrm{BV}$ at $30^{\circ} \mathrm{C}$ in glass cuvettes sealed with a rubber stopper and an aluminum cap under strictly anaerobic conditions. To make the conditions strictly anaerobic, the cuvette and the assay buffer (50mM Tris-HCl buffer $\mathrm{pH} 7.4$ containing $10 \mathrm{mM} \mathrm{BV}$ ) were flushed with a constant flow of $\mathrm{N}_{2}$ for 20 min. Reactions were started by adding the FDH sample to the cuvette and increase in absorbance of BV at $600 \mathrm{~nm}$

$\left(\varepsilon_{600 \mathrm{~nm}}=8.3 \mathrm{mM}^{-1} \mathrm{~cm}^{-1}\right)$ was monitored. Activity was expressed as units/mg of protein, where one unit $(\mathrm{U})$ is equivalent to oxidation of $1 \mu \mathrm{mol}$ formate/min.

\section{Results and Discussion}

\section{Recombinant and soluble EcFDH-O in E.coli}

184 We started with a feasibility study to determine whether EcFDH-O can be expressed as a soluble protein by deleting the membrane subunit, FdoI. An E.coli mutant with a defective fdoI gene (JW3863) was cultivated under microaerobic and aerobic conditions, together with the wild type strain (BW25113) and fdoG genedefective mutant (JW3865). Their activities were assessed by the in-gel activity stain method. Although none of the samples from aerobic cultures exhibited any active band (data not shown), all samples from microaerobic cultures resulted in the presence of a band at the top of the separation gel (Figure 4A, Lanes 1, 2 and 3). The band intensity in BW25113 was remarkably stronger than that in JW3863 or JW3865. One interpretation of this observation is that the strong band in BW25113 represents intact EcFDH-O and EcFDH-N, and the weak band in two mutants is due to the presence of only EcFDH-N. It is also likely that the intact EcFDH-O and EcFDH-N cannot go through the separation gel because of their insolubility and give rise to the active bands at the top of the gel. In accordance with this hypothesis, the weak band at the top of the gel disappeared in the BW $\Delta \Delta$ strain that harbors deletions in EcFDH-O and EcFDH-N (Figure 4A, Lane4). Interestingly, JW3865 showed a weak band with higher mobility ( $100 \mathrm{kDa}$ ) (Figure 4A, Lane2), suggesting that EcFDH-O can be expressed as a soluble heterodimeric protein consisting of FdoG and FdoH 
We next examined host-vector systems with $\mathrm{BW} \Delta \Delta$ strain as the host. The plasmids, pBR-fdoGHI, pBRfdoGHst, pBR-fdoGHtst, and pUC-fdoGHtst were introduced in $\mathrm{BW} \Delta \Delta$ and the resulting transformants were grown under microaerobic conditions. As shown in Lane 5 of Figure 4A, the transformant pBRfdoGHI/BW $\Delta \Delta$ presented a strong band at the same position as BW25113, indicating that the expression system can produce active recombinant EcFDH-O at the same level as the native system. pBRfdoGHst/BW $\Delta \Delta$ also showed a weak band similar to JW3863 (Figure 4A, Lane6), suggesting a successful production of soluble recombinant EcFdoGH protein. In $\mathrm{pBR}-\mathrm{fdoGHtst} / \mathrm{BW} \Delta \Delta$ that carries the membraneanchor truncated EcFdoGH (EcFdoGHt), no band was observed (data not shown). Protein activity was recovered in pUC-fdoGHtst/BW $\Delta \Delta$, which harbors the same gene and the same promoter as pBRfdoGHtst/BW $\Delta \Delta$, but the different plasmid (Figure 4A, Lane7). These observations indicated that EcFdoGH is destabilized by deletion of the membrane-anchor in EcFdoGHt, but the enhanced gene expression level in pUC-fdoGHtst/BW $\Delta \Delta$ due to high plasmid copy number results in the the increased accumulation of the recombinant protein in cell. Indeed, the quantitative PCR analysis revealed that the $f d o G$ transcript level in pUC-fdoGHtst/BW $\Delta \Delta$ was remarkably higher than that in BW25113 and pBR-fdoGHtst/BW $\Delta \Delta$ (Figure 5). Interestingly, the C-terminal-truncated protein, fdoGHtst, was less migrated than fdoGHst. But, it is reasonable because the C-terminal truncation results in the decrease in negative charges by 4 , which would significantly affect the mobility in non-denaturing polyacrylamide gel electrophoresis.

216 In spite of the high expression level, the activity of EcFdoGHt in pUC-fdoGHtst/BW $\Delta \Delta$ was much lower 217 than that of EcFDH-O in BW25113, indicating a significantly low stability of EcFdoGHt. To get improved 218 activity of EcFdoGHt, EcFdoGHt genes were subjected to random mutagenesis, and the resultant mutants 219 (ca. 500 clones) were randomly selected and assessed in 8-strip PCR tubes (Figure 4B). This resulted in the identification of one clone expressing FDH at a significantly higher level (Figure 4A, Lane8). The plasmid isolated from the clone was designated as pUC-fdoGHtst'. DNA sequencing analysis confirmed the presence of two mutations in pUC-fdoGHtst', one in the promoter region $(\mathrm{C} \rightarrow \mathrm{T}, 55$ bases upstream of the start codon) and another in the signal peptide cleavage sites $(\mathrm{T} \rightarrow \mathrm{C}$, Lue32 $\rightarrow$ Pro), suggesting that they may alter the 224 transcript level, the efficiency of the maturation processes or the protein stability. As shown in Figure 5, the 
it seemed to be insufficient to explain the result. To evaluate the effect of each mutation, we prepared two 227 plasmids, one carrying the single mutation in the promoter region (pUC-fdoGHtst'MW), and the other with 228 the mutation in the signal peptide cleavage site (pUC-fdoGHtst'WM). As shown in Figure 4C, BW $\Delta \Delta$ strain transformed with pUC-fdoGHtst'MW (pUC-fdoGHtst'MW/BW $\Delta \Delta$ ) exhibited a trace level of activity, whereas the pUC-fdoGHtst'WM transformant (pUC-fdoGHtst' ${ }^{\prime} \mathrm{WM} / \mathrm{BW} \Delta \Delta$ ) expressed significantly higher activity, clearly demonstrating that the mutation Lue $32 \rightarrow$ Pro has a crucial effect on the expression level of active FDH. Two plausible possibilities are: (1) the mutation at the signal peptide sites may retard the transportation of EcFdoGHt to periplasm membrane and/or cleavage of the signal peptide, which helps to complete the maturation process, (2) the mutation may prevent the signal peptide cleavage, which stabilizes the protein structure.

To purify the EcFdoGHt protein tagged with Strep-tagII, we applied the lysate of pUC-fdoGHtst'/BW $\Delta \Delta$ to StrepTactin-Sepharose column. However, a lot of active protein was found in the flow-through fractions (Figure 4D), raising the possibility that the Strep-tagII is not accessible for binding to the column. This may happen due to digestion of the C-terminal region, or its sequestration inside the protein. Interestingly, although the expression level of histidine-tagged FDH in pUC-fdoGHtHis'/BW $\Delta \Delta$ was almost the same as the Strep-tagII version in pUC-fdoGHtst'/BW $\Delta \Delta$ (Figure 4A, Lane 9 and 10), the purification yield of the histidine-tagged FDH was remarkably higher than the FDH with Strep-tagII (Figure 4D). The western blotting analysis demonstrated that the purified protein samples showed the expected immune bands (data not shown), but the purity and purification fold cannot be estimated because of the low amount of the purified FDH. The specific activity of partially purified EcFdoGHt with histidine tag was $0.2 \mathrm{U} / \mathrm{mg}$.

\section{Desulfovibrio FDH in E.coli}

248 It is known that Desulfovibrio species express soluble metal-dependent FDHs, but isolation of proteins from these anaerobic bacteria as hosts is time consuming and labor intensive. Therefore, we tried to use the tractable host, E.coli, to produce Desulfovibrio FDHs. All heterodimeric FDH genes identified in D. vulgaris (DvMF643-644, DvMF646-647, DvMF1217-1216 and DvMF2533-2534) and D. gigas (DgfdhA and DgfdhB) were cloned into pBR322 (pBR-DvMF643st, pBR-DvMF646st, pBR-DvMF1217st, pBR-DvMF2533st, and pBR-DgFDHst) together with the promoter of EcFDH-O (Figure 3). Unfortunately, BW $\Delta \Delta$ cells transformed 
with these plasmids showed no significant FDH activities under anaerobic, microaerobic, and aerobic conditions. Further attempts by deletion of Strep-tagII (pBR-DvMF643, pBR-DvMF646, pBR-DvMF1217, and pBR-DvMF2533), vector exchange to pUC (pUC-DvMF1217st and pUC-DgFDHst), and swapping the catalytic subunit of $D$. gigas FDH (DgfdhA) with $E$. coli fdoG (pUC-fdoG/DgigHst) exhibited no significant effects. These results indicated that Desulfovibrio FDH polypeptides of s cannot be adequately processed properly by E.coli maturation system. Therefore, we tried the co-expression of accessory genes from $D$. vulgaris and Desulfovibrio FDHs using pACYC-FdhDE1233, pACYC-FdhE645, and pACYC-FdhE648. However, no FDH activity was detected, indicating that the accessory genes we tried in this study are not enough to complete the maturation process of Desulfovibrio FDHs.

\section{Modification of Desulfovibrio FDH in Desulfovibrio}

One of the techniques for modification of the Desulfovibrio FDHs is gene manipulation by homologous recombination. We first optimized gene introduction method into D. vulgaris by electroporation and found that the highest efficiency (10-20 colonies per electroporation) was obtained by the following procedure: (1) D. vulgaris cells were washed twice with cold water, (2) plasmid DNA was added up to a final concentration of $20 \mathrm{ng} / \mathrm{ml}$, (3) electroporation voltage of $1750 \mathrm{~V}$ was charged, (4) the electroporated cells were cured for over $12 \mathrm{~h}$ under an $\mathrm{N}_{2}$ atmosphere, and (5) the cells were spread onto the selection medium containing 10 $\mu \mathrm{g} / \mathrm{ml}$ chloramphenicol. We then used quantitative PCR to show that the FDH composed of large and small subunits coded by $D v M F 1217$ and 1216, respectively, exhibited the highest expression level in four $D$. vulgaris FDHs (data not shown). Therefore, we chose C-terminus of small subunit of FDH (DvMF1216) as the insertion site and constructed two recombination vectors, pDvMF-cmSt and pDvMF-cmPsaE (Figure 3). pDvMF-cmSt was designed to isolate a strain producing a soluble dimeric FDH with Strep-tagII (DvFDHst) by direct marker exchange (without the isolation step for single recombinants). On the other hand, pDvMFcmPsaE was designed to isolate a strain generating a FDH fused with PsaE, one of the subunits of PSI complex (DvFDH-PsaE) by direct marker exchange. The two plasmids were then introduced into cells by the optimized electroporation conditions, yielding 81 independent clones. From these clones, a recombinant derived from pDvMF-cmPsaE was successfully isolated, but no recombinant from pDvMF-cmSt could be 
(18 of 81 clones) were unknown chloramphenicol-tolerant strains, indicating that the simultaneous double cross-over recombination event was rather rare (1 in 81 clones).

284 To introduce a selection pressure for the second recombination, the sucrose dependent lethal gene, $\operatorname{sacB}$, was inserted into pDvMF-cmSt, generating pDvMF-cmSt' (Figure 3). pDvMF-cmSt' was introduced into the $D$. vulgaris cells by electroporation, and then dozens of the single recombinant clones were isolated, in which the plasmid was incorporated into the genome. Several of these clones were grown in the presence of $10 \%$ sucrose to select for the deletion of $s a c B$ gene by second recombination. Of the resultant sucrose-tolerant clones, 61 clones were investigated for the genome sequence around the insertion site, revealing that only one clone possessed the insertion mutation of the Strep-tagII sequence into the designed site and others having partially deleted the $s a c B$ gene.

For the protein extraction from $D$. vulgaris cells, we optimized the extraction conditions. D. vulgaris cells are surrounded by black iron sulfide powder, which protect against cell disruptions. Although sonication treatment is usually used for the disruption of $D$. vulgaris cells, we found that the FDH activity is easily inactivated by sonication. We, therefore, employed the lysozyme-DNase treatment for the cell lysis. The supernatant containing soluble FDHs was prepared by centrifuging the lysate in the presence of $0.1 \%$ Triton $\mathrm{X}-100$. The addition of Triton $\mathrm{X}-100$ was not indispensable in this procedure, but had an effect to stabilize the active form of FDH, improving the yield.

The cell lysates prepared as described above were analyzed by western blotting assay. As shown in Figure $6 \mathrm{~A}$, a band with an expected molecular mass $(\sim 30 \mathrm{kDa})$ on the western blotting was observed in DvFDHst sample, indicating that DvFDHst was successfully produced, but no band was seen for pDvMF-cmPsaE (data not shown). The supernatants of the lysate samples were subjected to the in-gel activity assay, and showed several active bands, which is due to the presence of several FDH isozymes and makes it difficult to assess the expression of DvFDHst and DvFDH-PsaE (Figure 6B). DvFDHst and DvFDH-PsaE were isolated on StrepTactin beads from the lysate supernatants, and stained by incubating in the presence of $2 \mathrm{mM} \mathrm{BV}, 2$ $\mathrm{mM}$ nitroblue tetrazolium, $80 \mathrm{mM}$ formate, and $0.1 \%$ Triton $\mathrm{X}-100$ under a nitrogen atmosphere at $30^{\circ} \mathrm{C}$. As shown in Figure 6C, DvFDHst on StrepTactin beads was remarkably stained, whereas DvFDH-PsaE sample was not obviously stained. These results indicated that the soluble recombinant DvFDHst can be expressed 
as an active form in the D. vulgaris recombinant strain, and successfully isolated from the lysate using

StrepTactin beads, and that the expression of DvFDH-PsaE is controversial.

\section{Conclusions}

We successfully showed that EcFdoGHt tagged with histidine tag can be expressed in E. coli and purified.

The specific activity of partially purified EcFdoGHt $(0.2 \mathrm{U} / \mathrm{mg})$ was relatively lower than that of native

FDH. However, we demonstrated the first example of the improvement of metal-dependent FDHs by a

directed evolution procedure, which will open the door to engineering studies on FDHs. The attempts to express Desulfovibrio FDHs in E. coli were hampered by the lack of knowledge of the maturation processes.

We should study the prerequisites for the complete maturation of metal-dependent FDHs. Generation of the recombinant FDH by homologous recombination was also intractable because of the difficulty in the isolation of the recombinants. However, we succeeded in the preparation of the soluble DvFDH tagged with

Strep-tagII, and successfully isolated using StrepTactin beads. Our demonstration of the preparation of tagged DvFDH is especially important in the development of FDH electrode.

\section{ACKNOWLEDGMENTS}

This research was supported by JST PRESTO “Light Energy and Chemical Coversion”.

\section{References}

[1] Ferry JG. Formate Dehydrogenase. Fems Microbiol Lett. 1990;87:377-82.

[2] Tishkov VI, Popov VO. Protein engineering of formate dehydrogenase. Biomol Eng. 330 2006;23:89-110.

[3] Bassegoda A, Madden C, Wakerley DW, Reisner E, Hirst J. Reversible Interconversion of $\mathrm{CO} 2$ and Formate by a Molybdenum-Containing Formate Dehydrogenase. J Am Chem Soc. 2014;136:15473-6.

[4] Reda T, Plugge CM, Abram NJ, Hirst J. Reversible interconversion of carbon dioxide and formate by an electroactive enzyme. P Natl Acad Sci USA. 2008;105:10654-8.

[5] Leopoldini M, Chiodo SG, Toscano M, Russo N. Reaction Mechanism of Molybdoenzyme Formate Dehydrogenase. Chem-Eur J. 2008;14:8674-81.

[6] Ihara M, Kawano Y, Urano M, Okabe A. Light Driven CO2 Fixation by Using 340 Cyanobacterial Photosystem I and NADPH-Dependent Formate Dehydrogenase. Plos One. 2013;8. 
[7] Boyington JC, Gladyshev VN, Khangulov SV, Stadtman TC, Sun PD. Crystal structure of formate dehydrogenase $\mathrm{H}$ : Catalysis involving Mo, molybdopterin, selenocysteine, and an Fe4S4 cluster. Science. 1997;275:1305-8. [8] Teixeira S, Dias JM, Carvalho AL, Bourenkov G, Bartunik H, Almendra MJ, et al. Crystallographic studies on a tungsten-containning formate dehydrogenase from Desulfovibrio gigas. J Inorg Biochem. 1999;74:89-.

[9] Raaijmakers H, Macieira S, Dias JM, Teixeira S, Bursakov S, Huber R, et al. Gene sequence and the 1.8 angstrom crystal structure of the tungsten-containing formate dehydrogenase from Desulfovibrio gigas. Structure. 2002;10:1261-72.

[10] Jormakka M, Tornroth S, Abramson J, Byrne B, Iwata S. Purification and crystallization of the respiratory complex formate dehydrogenase-N from Escherichia coli. Acta Crystallogr D. 2002;58:160-2.

[11] Gladyshev VN, Khangulov SV, Axley MJ, Stadtman TC. Coordination of Selenium to Molybdenum in Formate Dehydrogenase-H from Escherichia-Coli. P Natl Acad Sci USA. 1994;91:7708-11.

[12] Axley MJ, Grahame DA. Kinetics for Formate Dehydrogenase of Escherichia-Coli Formate-Hydrogenlyase. J Biol Chem. 1991;266:13731-6.

[13] Axley MJ, Grahame DA, Stadtman TC. Escherichia-Coli Formate-Hydrogen Lyase Purification and Properties of the Selenium-Dependent Formate Dehydrogenase Component. J Biol Chem. 1990;265:18213-8.

[14] Berg BL, Stewart V. Structural Genes for Nitrate-Inducible Formate Dehydrogenase in Escherichia-Coli K-12. Genetics. 1990;125:691-702.

[15] Abaibou H, Pommier J, Benoit S, Giordano G, Mandrandberthelot MA. Expression and Characterization of the Escherichia-Coli Fdo Locus and a Possible Physiological-Role for Aerobic Formate Dehydrogenase. J Bacteriol. 1995;177:7141-9.

[16] Benoit S, Abaibou H, Mandrand-Berthelot MA. Topological analysis of the aerobic membrane-bound formate dehydrogenase of Escherichia coli. J Bacteriol. 1998;180:662534.

[17] Costa C, Teixeira M, LeGall J, Moura JJG, Moura I. Formate dehydrogenase from Desulfovibrio desulfuricans ATCC 27774: Isolation and spectroscopic characterization of the active sites (heme, iron-sulfur centers and molybdenum). J Biol Inorg Chem. 1997;2:198-208.

[18] Sebban C, Blanchard L, Bruschi M, Guerlesquin F. Purification and Characterization of the Formate Dehydrogenase from Desulfovibrio-Vulgaris Hildenborough. Fems Microbiol Lett. 1995;133:143-9.

[19] Brondino CD, Passeggi MCG, Caldeira J, Almendra MJ, Feio MJ, Moura JJG, et al. Incorporation of either molybdenum or tungsten into formate dehydrogenase from Desulfovibrio alaskensis NCIMB 13491; EPR assignment of the proximal iron-sulfur cluster to the pterin cofactor in formate dehydrogenases from sulfate-reducing bacteria. J Biol Inorg Chem. 2004;9:145-51.

[20] da Silva SM, Pimentel C, Valente FMA, Rodrigues-Pousada C, Pereira IAC. Tungsten and Molybdenum Regulation of Formate Dehydrogenase Expression in Desulfovibrio vulgaris Hildenborough. J Bacteriol. 2011;193:2909-16.

[21] Ihara M, Nakamoto H, Kamachi T, Okura I, Maeda M. Photoinduced hydrogen production by direct electron transfer from photosystem I cross-linked with cytochrome c(3) to [NiFe]-hydrogenase. Photochem Photobiol. 2006;82:1677-85.

[22] Link AJ, Phillips D, Church GM. Methods for generating precise deletions and insertions in the genome of wild-type Escherichia coli: Application to open reading frame characterization. J Bacteriol. 1997;179:6228-37. 


\section{Table 1, Strains and Plasmids}

\begin{tabular}{|c|c|}
\hline Strains & Genotype \\
\hline D. gigas & DSM1382 \\
\hline D. vulgaris & DSM19637 \\
\hline $1216 s t$ & strep-tagged DvMF1217, chloramphenicol resistant cassette \\
\hline 1216-PsaEst & PsaE-fused DvMF1217, chloramphenicol resistant cassette \\
\hline \multicolumn{2}{|l|}{ BW25113 } \\
\hline JW3865 & BW25113 $\Delta$ fdoG::KanR \\
\hline JW3863 & BW25113 $\Delta$ fdol::KanR \\
\hline $\mathrm{BW} \Delta \Delta$ & BW25113 $\Delta f d o G H I, \Delta f d n G H I$ \\
\hline \multicolumn{2}{|l|}{ Plasmids } \\
\hline pKO- $\Delta \mathrm{fdnGHI}$ & E. coli fdnG fragment and $E$. coli fdnI fragment in pKO3 \\
\hline pKO- $\Delta$ fdoGHI & E. coli fdoG fragment and $E$. coli fdol fragment in pKO3 \\
\hline pBR-fdoGHI & E. coli fdo Promoter, E. coli fdoG, E. coli fdoH and E. coli fdol in pBR322 \\
\hline pBR-fdoGH & E. coli fdo Promoter, E. coli fdoG and E. coli fdoH in pBR322 \\
\hline pBR-fdoGHst & E. coli fdo Promoter, E. coli fdoG and strep-tagged E. coli fdoH in pBR322 \\
\hline pBR-fdoGHtst & $\begin{array}{l}\text { E. coli fdo Promoter, E. coli fdoG and membrane anchor-truncated and } \\
\text { strep-tagged E. coli fdoH in pBR322 }\end{array}$ \\
\hline pUC-fdoGHtst & $\begin{array}{l}\text { E. coli fdo Promoter, E. coli fdoG and membrane anchor-truncated and } \\
\text { strep-tagged E. coli fdoH in pUC19 }\end{array}$ \\
\hline pUC-fdoGHtHis & $\begin{array}{l}\text { E. coli fdo Promoter, E. coli fdoG and membrane anchor-truncated and His- } \\
\text { tagged } E \text {. coli fdoH in pUC19 }\end{array}$ \\
\hline pBR-DvMF643 & E. coli fdo Promoter, DvMF643 and DvMF644 in pBR322 \\
\hline pBR-DvMF646 & E. coli fdo Promoter, DvMF646 and DvMF647 in pBR322 \\
\hline pBR-DvMF1217 & E. coli fdo Promoter, DvMF1216 and DvMF1217 in pBR322 \\
\hline pBR-DvMF2533 & E. coli fdo Promoter, DvMF2533 and DvMF2534 in pBR322 \\
\hline pBR-DvMF643st & E. coli fdo Promoter, DvMF643 and strep-tagged DvMF644 in pBR322 \\
\hline pBR-DvMF646st & E. coli fdo Promoter, DvMF646 and strep-tagged DvMF647 in pBR322 \\
\hline pBR-DvMF1217st & E. coli fdo Promoter, DvMF1216 and strep-tagged DvMF1217 in pBR322 \\
\hline pBR-DvMF2533st & E. coli fdo Promoter, DvMF2533 and strep-tagged DvMF2534 in pBR322 \\
\hline pBR-DgFDH & E. coli fdo Promoter, DgfdhA and DgfdhB in pBR322 \\
\hline pUC-DvMF1217st & E. coli fdo Promoter, DvMF1216 and strep-tagged DvMF1217 in pUC19 \\
\hline pUC-DgFDHst & E. coli fdo Promoter, DgfdhA and DgfdhB in pUC19 \\
\hline pUC-fdoGH/Dgig & $\begin{array}{l}\text { E. coli fdo Promoter, DgfdhA and chemera gene of } E \text {. coli fdoH and DgfdhB } \\
\text { in pUC19 }\end{array}$ \\
\hline pACYC-FdhDE1233 & E. coli fdo Promoter, DvMF1232(FdhD) and DvMF1233(fdhE) in pACYC184 \\
\hline pACYC-FdhE645 & E. coli fdo Promoter and DvMF645 in pACYC184 \\
\hline pACYC-FdhE648 & E. coli fdo Promoter and DvMF648(FdhE) in pACYC184 \\
\hline pDvMF-cmSt & $\begin{array}{l}\text { DvMF1215 }(M o b B) \text { fragment, strep-tagged } D v M F 1216, D v M F 1217 \\
\text { fragment, chloramphenicol resistant cassette and } D v M F 1216 \text { promoter in } \\
\text { pUC19 }\end{array}$ \\
\hline pDvMF-cmSt' & $S a c B$ in pDvMF-cmSt \\
\hline pDvMF-cmPsaE & $\begin{array}{l}\text { DvMF1215(MobB) fragment, PsaE-fused DvMF1216, DvMF1217 fragment, } \\
\text { chloramphenicol resistant cassette and DvMF1216 promoter in pUC19 }\end{array}$ \\
\hline
\end{tabular}


Table 2, Primer sequences

\section{Primer}

set

1

2

3

4

5
5'-CCGGGGATCGCGGCCAAGATCTGCGCTGGCG-3'

5'-ATACCGTGCGATCGTCGTCAACGAGG-3'

5'-CCGGGGATCGCGGCCTGGACGTCAGTCGCAGAC-3'

5'-GCGCTACCCAAGTGGCGGATGTCGG-3'

5'-CTTTCGTCTTCAAGAATTCGTTACAAACCCTTCCTGG-3'

5'-CTTTCGTCTTCAAGAATTCGTTACAAACCCTTCCTGG-3'

5' -CTTTCGTCTTCAAGAATTCGTTACAAACCCTTCCTGG-3'

5'-CTTTCGTCTTCAAGAATTCGTTACAAACCCTTCCTGG-3'

5'-TGCGTTGCGCTCACTGCC-3'

5'-AGTGAGCGCAACGCAGTTACAAACCCTTCCTGG-3'

5'-GAATCCAGATCTCACCACCACCACCACCACTAATTAAG-3'

5'-CTTTCGTCTTCAAGAATTCGTTACAAACCCTTCCTGG-3'

5'-CAAGGAGCAATCCATGCGAAGACGCCAA-3'

5'-CAAGGAGCAATCCATGAACACTACGCGGC-3'

5'-CAAGGAGCAATCCATGACTGTCAATCGCAGG-3'

5'-CAAGGAGCAATCCATGAAAATGAACCGGC-3'

5'-CAAGGAGCAATCCATGCGAAGACGCCAA-3'

5'-CAAGGAGCAATCCATGAACACTACGCGGC-3'

5'-CAAGGAGCAATCCATGACTGTCAATCGCAGG-3'

5'-CAAGGAGCAATCCATGAAAATGAACCGGC-3' )

5'-CAAGGAGCAATCCATGCTGATCAAACGTCG-3'

5'-CAAGGAGCAATCCATGCTGATCAAACGTCG-3'

5'-AGTGAGCGCAACGCAGTTACAAACCCTTCCTGG-3'

5'-AGTGAGCGCAACGCAGTTACAAACCCTTCCTGG-3'

5'-TAACGGAGACGACATATGTCCAAAGGCTTCTTCG-3'

5'-GAAACAAGGAGCAATCCATGCCCGACGCTGCC-3'

5'-GAAACAAGGAGCAATCCATGGCCGCCAAACACC-3'

5'-GAAACAAGGAGCAATCCATGACCTTCGATTTCGACC-3' 5' -TGATTACGCCAAGCTATGGGAAAGATGTTCTTCATC-3'

5'-TGGAGCCACCCGCAGTTCGAAAAATAGCGCTGATGTCC GGCG-3'

5'-CAGGGCGGGGCGTAAGAGAGTAATGCTGTCGTG-3'

5'-GGAAAGGGGACTACCCGTAGAACCGCATTGCAAC-3'

5'-TGATTACGCCAAGCTATGGgAAAGATGTTCTTCATC-3'

5'-GGAGGTTCTGGAGGTGTACAACGTG-3'

5'-TAGGGTCGACTCTAGCTGCCGTTCACTATTATTTAG-3'

Sequence

5'-GACGACGATCGCACGGTATTCCGGAAAC-3'

5'-TAGAGGATCGCGGCCGCGCTGGTAACCCATCC-3'

5'-CCGCCACTTGGGTAGCGCAGACGGT-3'

5'-TAGAGGATCGCGGCCGCCGCTTCTGCCTTCTCG-3'

5'-CAAGGGCATCGGTCGACTGCGAATACTCATTCAGC-3'

5'-CAAGGGCATCGGTCGACTCATTTGCGCTCCTC-3'

5'-CAAGGGCATCGGTCGACTTATTTTTCGAACTGCGGGTGG CTCCAAGCAGATTTGCGCTCCTCGTC-3'

5'-CAAGGGCATCGGTCGACTTATTTTTCGAACTGCGGGTGG CTCCAAGCAGATTCAGCTTTCTTTTCCG-3'

5'-TCTGCTTGGAGCCACCCGCAGTTCGAAAAATAATTAAGC CAGCCCCGA-3'

5'-GTGGCTCCAAGCAGATTCCGGGTTCTCCGGC-3'

5'-GGTGGTGAGATCTGGATTCCGGGTTCTCCGGC-3'

5'-CATGGATTGCTCCTTGTTTC-3'

5'-CAAGGGCATCGGTCGACCTACATGAAACCGGCG-3'

5'-CAAGGGCATCGGTCGACTTACACGCCCGCCGC-3'

5'-CAAGGGCATCGGTCGACCTACGCGCGCGCCCG-3'

5'-CAAGGGCATCGGTCGACCTACCCCGTGCGCAG-3'

5'-CAAGGGCATCGGTCGACTTATTTTTCGAACTGCGGGTG GCTCCAAGCAGACACGCCCGCCGCGTA-3'

5'-CAAGGGCATCGGTCGACTTATTTTTCGAACTGCGGGTGG CTCCAAGCAGACATGAAACCGGCGTA-3'

5'-CAAGGGCATCGGTCGACTTATTTTTCGAACTGCGGGTGG CTCCAAGCAGACGCGCGCGCCCGGAA-3'

5'-CAAGGGCATCGGTCGACTTATTTTTCGAACTGCGGGTGG CTCCAAGCAGACCCCGTGCGCAGCGG-3'

5' -CAAGGGCATCGGTCGACNGCCACNGCGTATTCG-3'

5'-CAAGGGCATCGGTCGACTTATTTTTCGAACTGCGGGTGG CTCCAAGCAGACNGCCACNGCGTATTCG-3'

5'-GCGGGTGGCTCCAAGCAGA-3'

5'-ATGTCGTCTCCGTTACACCTTTTCC-3'

5'-GCGGGTGGCTCCAAGCAGA-3'

5'-GTTATTGGTGCGTCGACTCAGGCTGAGGTTTTTGCG-3' 5'-GTTATTGGTGCGTCGACTCAATACATATTCCAGAAGGAA GG-3'

5'-GTTATTGGTGCGTCGACTCATTCCAGTACCCTGCC-3'

5'-CTGCGGGTGGCTCCACACCGCGTGCTTGAAGTAG-3'

5'-TTACGCCCCGCCCTGCCACTC-3'

5'-GGTAGTCCCCTTTCCCTG-3'

5'-CCTCTAGAGTCGACCCTACTCCGCAAGGCGG-3'

5'-ACCTCCAGAACCTCCCACCGCGTGCTTGAAGTAG-3'

5' -CTGCGGGTGGCTCCAGGATTTACCTTTTTTGGGGGG-3'

5'-CGgCCAGTGAATTCGTTATTTGTTAACTGTTAATTG-3' 

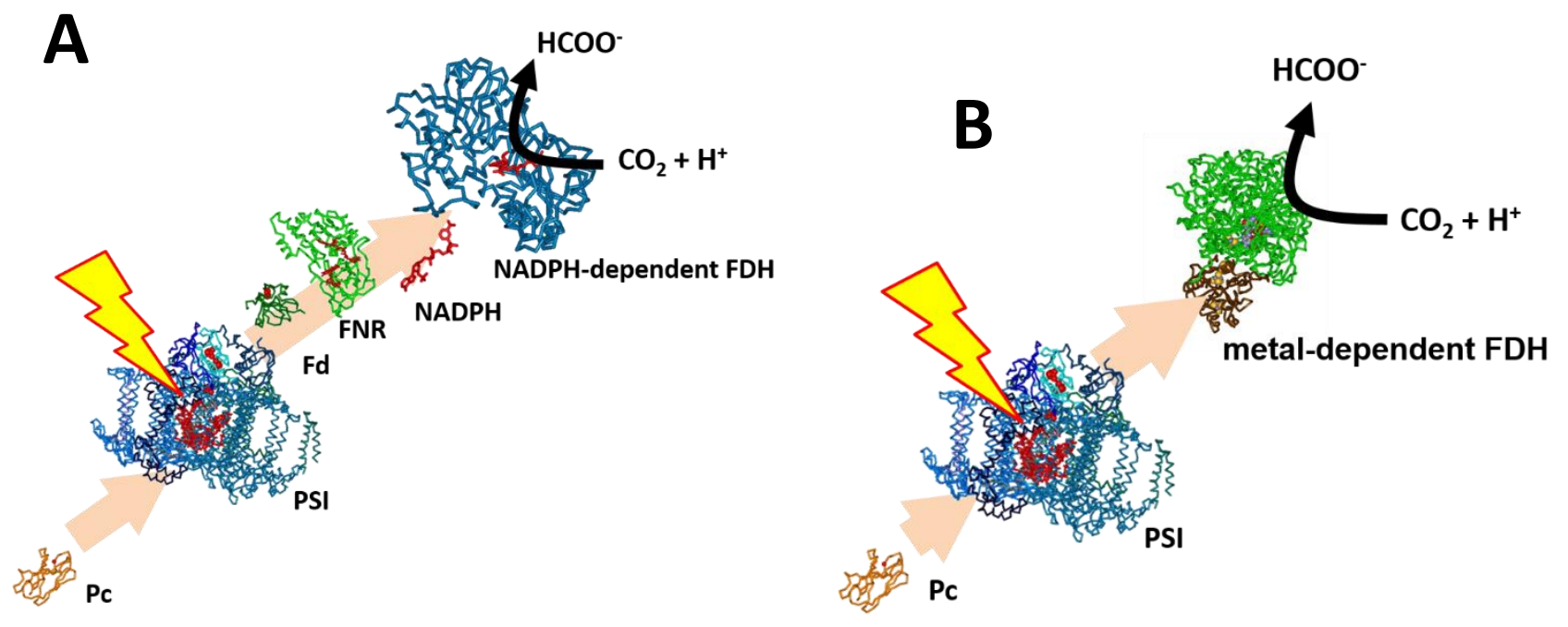

Figure 1. (A) The artificial light-driven carbon dioxide reduction system using NADPH-dependent FDH and photo-electron relay system consisting of plastocyanin $(\mathrm{Pc}), \mathrm{PSI}$, ferredoxin $(\mathrm{Fd})$, ferredoxin-NADP ${ }^{+}$ reductase (FNR) and NADP(H). (B) An example of direct light-driven carbon dioxide reduction using metal-dependent FDH and PSI. 

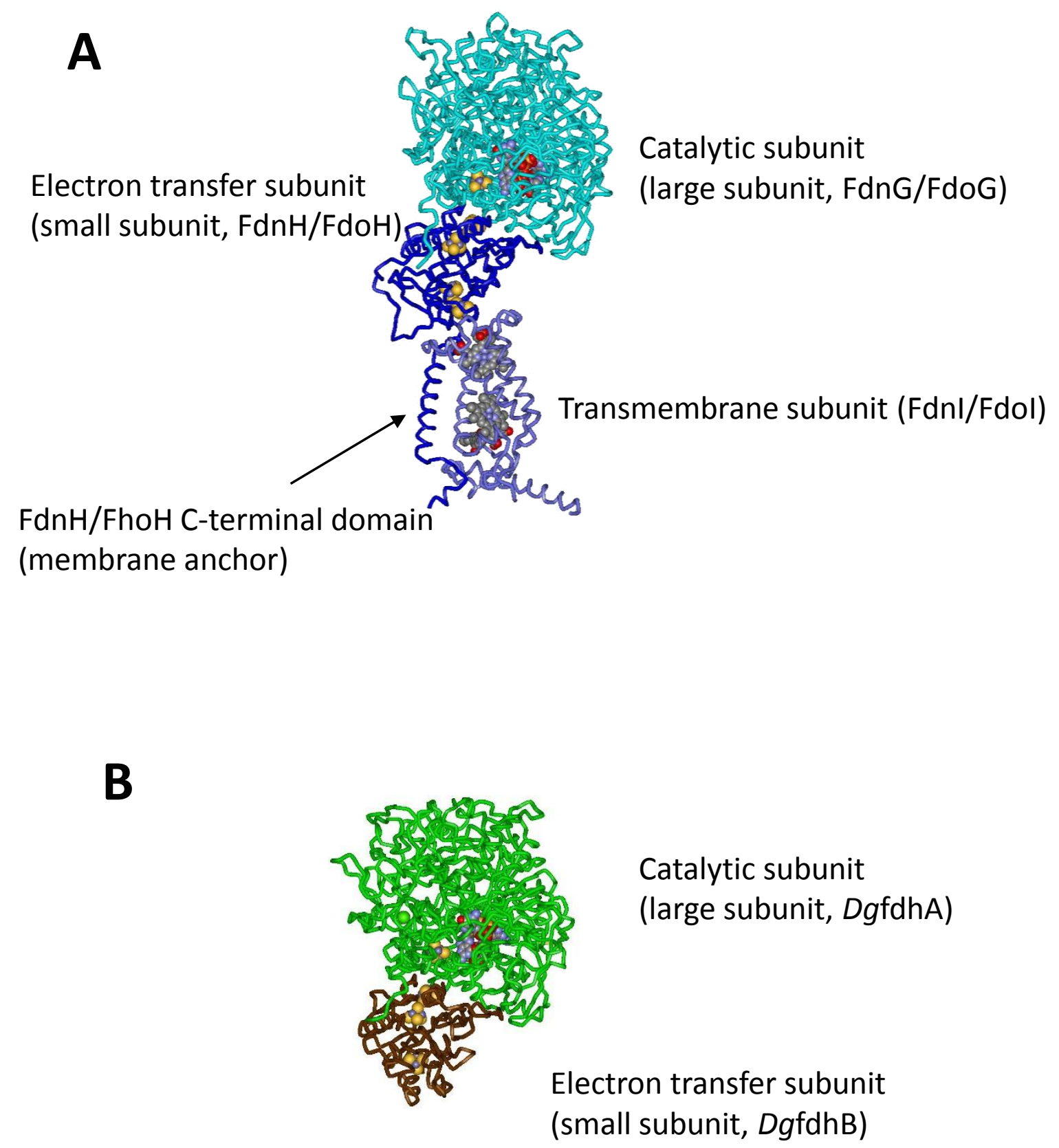

Figure 2. (A) Crystal structure of E. coli FDH-N (Jormakka, Tornroth et al. 2002). FDH-O exhibits high homology with FDH-N, and they are thought to have essentially the same structure. E. coli FDH-N and FDH-O contain a catalytic subunit, an electron transfer subunit and a transmembrane subunit. $E$. coli $\mathrm{FDHs}$ are tethered to membrane via the $\mathrm{C}$-terminal domain of the electron transfer subunit and the transmembrane subunit. (B) Crystal structure of $D$. gigas FDH (Teixeira, Dias et al. 1999). D. gigas FDH is composed of a catalytic subunit and an electron transfer subunit. 


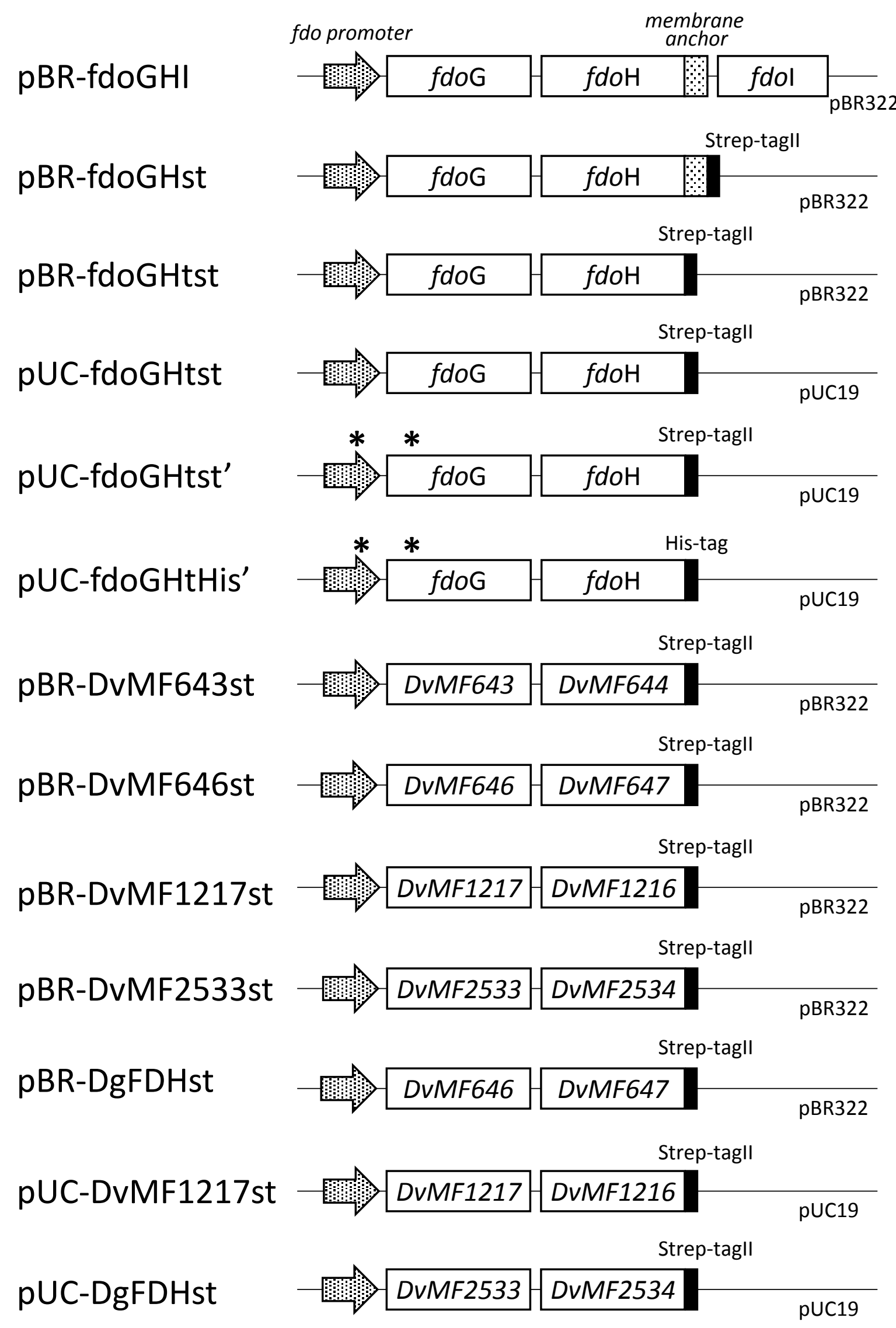

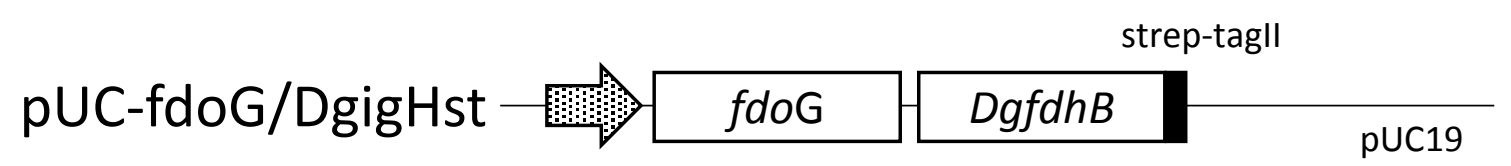


Figure 3. Schematic presentation of plasmid constructs. Open squares represent genes or gene fragments. The orientation of $f d o$ promoter and Dv1216 promoter are given by arrows. Strep-tagII and histidine tag are marked by closed squares. The asterisks indicate two mutation sites, 55 bases upstream of the start codon $(\mathrm{C} \rightarrow \mathrm{T})$ and the signal peptide cleavage sites $(\mathrm{T} \rightarrow \mathrm{C}$, Lue $32 \rightarrow$ Pro $)$. 

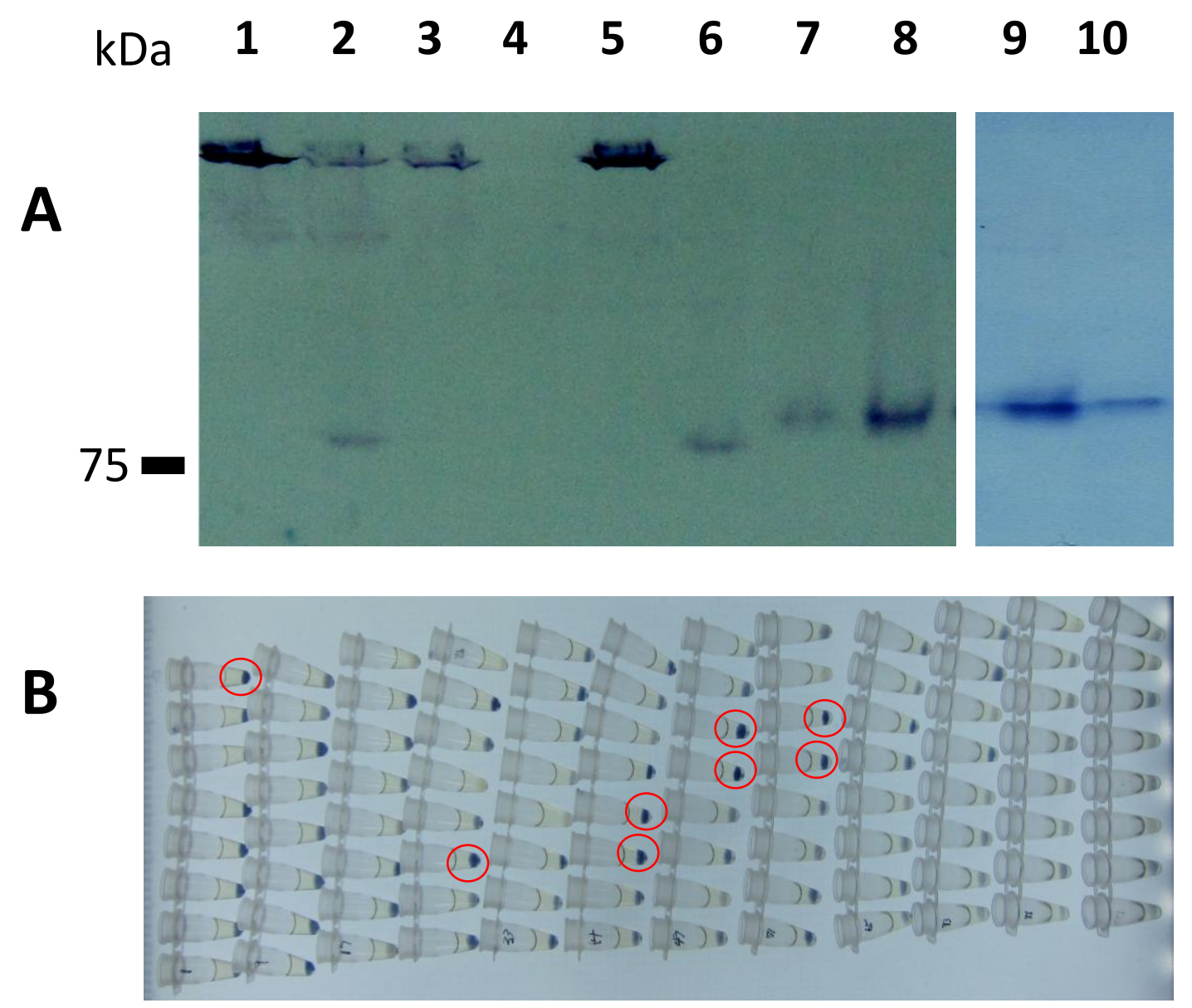

\section{3}
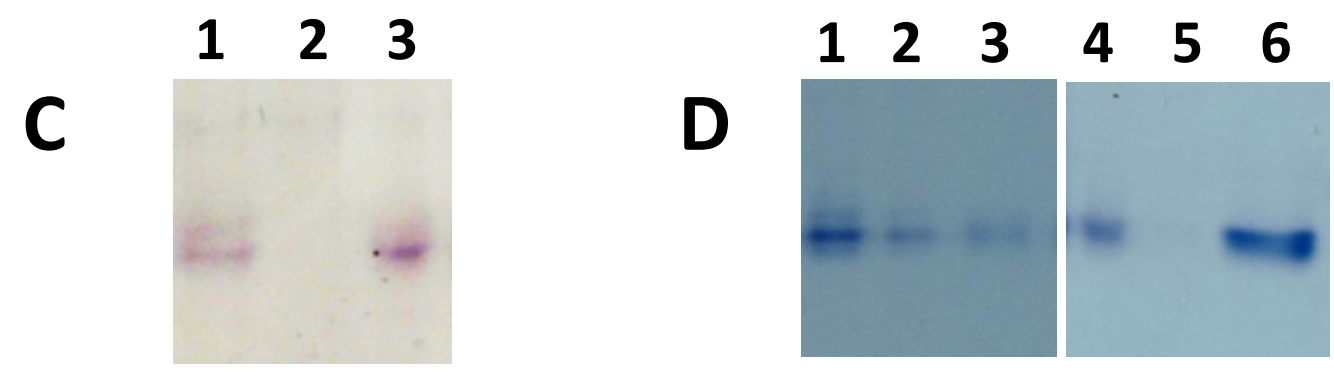

Figure 4. (A) In-gel activity stain analysis. The lysates $(10 \mu \mathrm{l}$ of $40 \mathrm{mg}$ wet cell pellet/ml in each well) from BW25113 (Lane 1), JW3863 (Lane 2), JW3865 (Lane 3), BW $\Delta \Delta$ (Lane4), pBR-fdoGHI/BW $\Delta \Delta$ (Lane 5), pBR-fdoGHst/BW $\Delta \Delta$ (Lane 6), pUC-fdoGHtst/BW $\Delta \Delta$ (Lane 7), pUC-fdoGHtst'/BW $\Delta \Delta$ (Lane 8 and 9) and pUC-fdoGHtHis'/BW $\Delta \Delta$ (Lane 10) were electrophoresed in a nondenaturing polyacrylamide gel and stained by incubating in the presence of $2 \mathrm{mM}$ BV, $2 \mathrm{mM}$ Nitrobule tetrazolium, $80 \mathrm{mM}$ formate, and $0.1 \%$ Triton X-100 under a nitrogen atmosphere at $30^{\circ} \mathrm{C}$. Only a $75 \mathrm{kD}$ band in molecular weight markers was identified. Pictures of Lanes 1-8 and Lane 9 and 10 were obtained from two independent experiments. (B) Screening for the highly active clones on StrepTactin-bead in 8-strip PCR tubes. Circles indicate the candidates with highly active clones. (C) In-gel activity stain analysis. The lysates from pUCfdoGHtst'/BW $\Delta \Delta$ (Lane 1), pUC-fdoGHtst'MW/BW $\Delta \Delta$ (Lane 2) and pUCfdoGHtst'WM/BW $\Delta \Delta$ were analyzed. (D) In-gel activity stain analysis of the supernatant (Lane 1), flow-through (Lane 2) and eluate (Lane 3) of pUCfdoGHtst'/BW $\Delta \Delta$ and supernatant (Lane 4), flow-through (Lane 5) and eluate (Lane 6) of pUC-fdoGHtHis'/BW $\Delta \Delta$. 


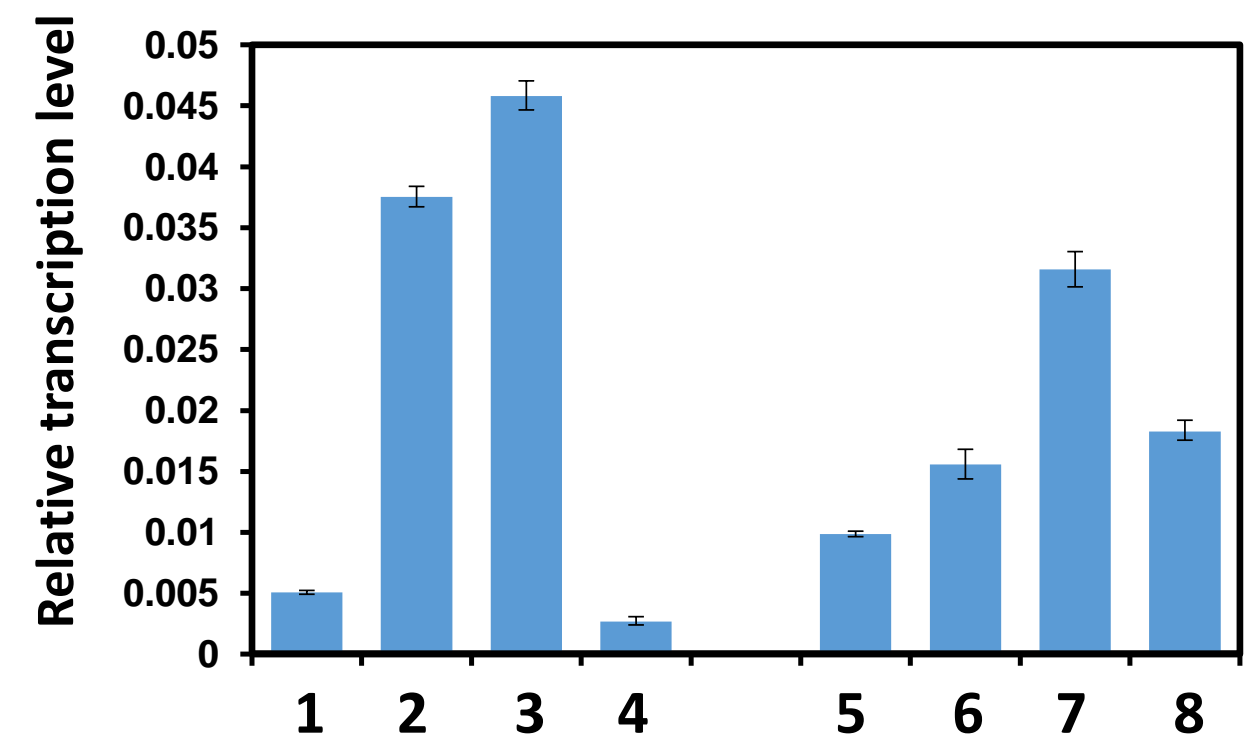

Figure 5. Relative transcript levels of $f d o G$ gene against internal standard gene 16srRNA. BW25113 (1 and 5), pUC-fdoGHtst'/BW $\Delta \Delta$ (2 and 6), pUCfdoGHtst/BW $\Delta \Delta$ (3 and 7), and pBR-fdoGHtst/BW $\Delta \Delta$ (4 and 8) were cultivated under microaerobic (1-4) and aerobic (5-8) conditions, and subjected to RNA extraction and quantitative PCR analysis. 


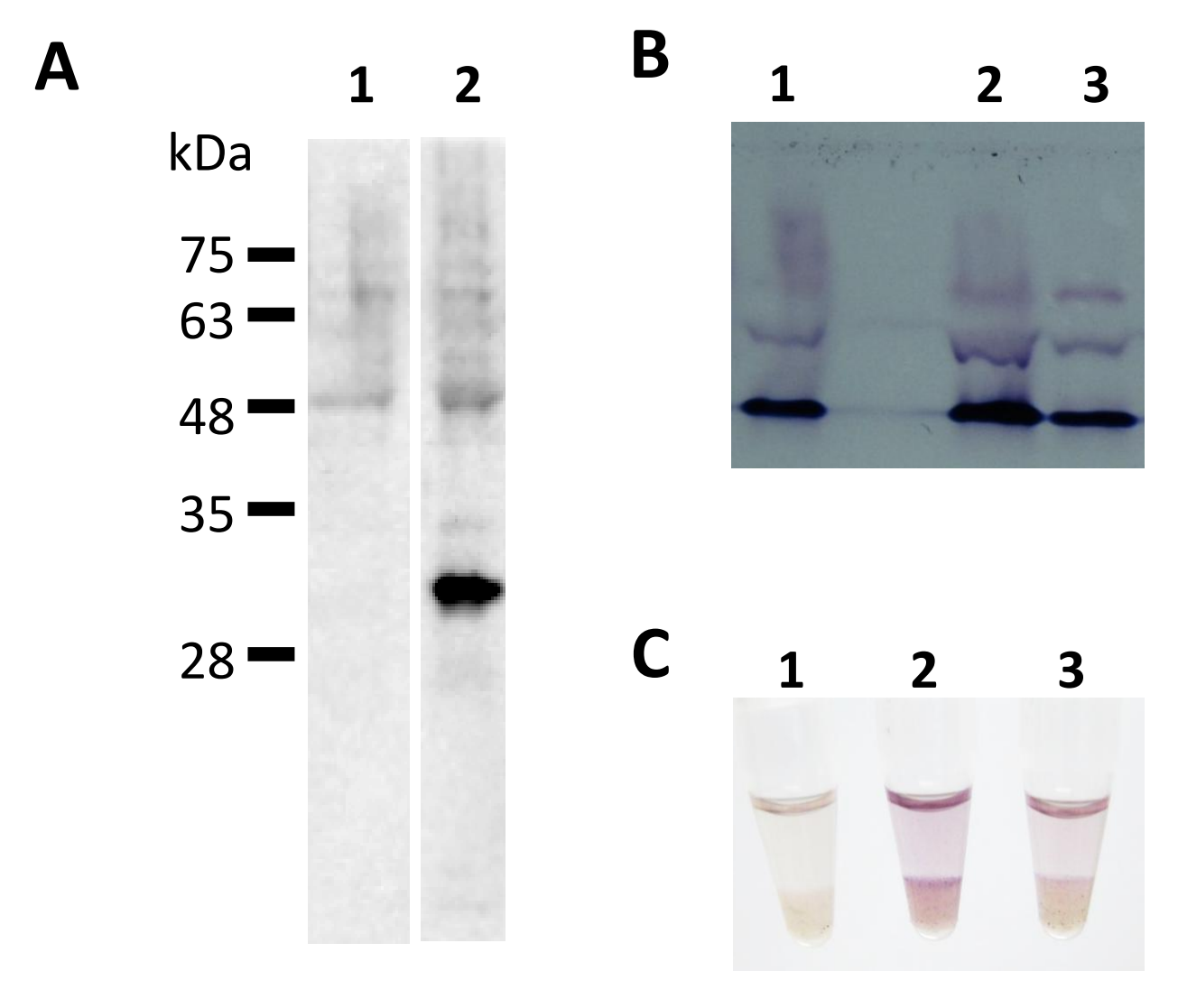

Figure 6. (A) Western blotting analysis. The lysates from wild type $D v \mathrm{MF}$ strain (Lane 1) and the recombinant strain from pDvMF-cmSt (Lane 2) were analyzed.

(B) In-gel activity stain analysis. The supernatants of the lysates from wild type $D v \mathrm{MF}$ strain (Lane 1), the recombinant strain from pDvMF-cmSt (Lane 2) and the recombinant strain from pDvMF-cmPsaE (Lane 3) were separated on a nondenaturing polyacrylamide gel and stained. (C) Activity stain on StreptTctin-beads in PCR tubes. The StrepTactin-beads were incubated with the supernatants of the lysate from wild type $D v \mathrm{MF}$ strain (Tube 1), the recombinant strain from pDvMFcmSt (Tube 2) and the recombinant strain from pDvMF-cmPsaE (Tube 3), washed and stained with formate, BV and nitroblue under nitrogen gas. 